\title{
Text Document Classification: AN APPROACH BASED ON INDEXING
}

\author{
B S Harish ${ }^{1}$, S Manjunath ${ }^{2}$ and D S Guru ${ }^{3}$ \\ ${ }^{1}$ Department of Information Science and Engineering, SJCE, Mysore \\ bsharish@ymail.com \\ ${ }^{2}$ JSS College of Arts, Commerce and Science, Mysore \\ manju_uom@yahoo.co.in \\ ${ }^{3}$ Department of Studies in Computer Science, University of Mysore \\ dsg@compsci.uni-mysore.ac.in
}

\begin{abstract}
In this paper we propose a new method of classifying text documents. Unlike conventional vector space models, the proposed method preserves the sequence of term occurrence in a document. The term sequence is effectively preserved with the help of a novel datastructure called 'Status Matrix'. Further the corresponding classification technique has been proposed for efficient classification of text documents. In addition, in order to avoid sequential matching during classification, we propose to index the terms in Btree, an efficient index scheme. Each term in B-tree is associated with a list of class labels of those documents which contain the term. Further the corresponding classification technique has been proposed. To corroborate the efficacy of the proposed representation and status matrix based classification, we have conducted extensive experiments on various datasets.
\end{abstract}

\section{KEYWORDS}

Text documents, Representation, Term sequence, Status Matrix, B-Tree, Classification

\section{INTRODUCTION}

Text documents have become the most common container of information. This is due to the increased popularity of the internet, emails, newsgroup messages etc, where text is the dominant type of information exchange. Nowadays many real time text mining applications have received a lot of research attention. Some of the applications are: spam filtering, emails categorization, directory maintenance, ontology mapping, document retrieval, routing, filtering etc $[1,2,3]$. Here, each application may handle million or even billions of text documents. Also World Wide Web (WWW) itself contains a large amount of documents, conference materials, publications, journals, editorials, news and information etc., available in electronic form. The available documents are in various forms and the information in them are not in organized form. The lack of organization of materials in the WWW motivates people to automatically manage the huge amount of information. This requires implementation of sophisticated learning agents that are capable of classifying relevant information and thereby increases the text organization over WWW [3].

From several decades, automatic document management tasks have gained a prominent status in the field of information retrieval. Until late 80's, text classification task was based on Knowledge Engineering (KE), where a set of rules were defined manually to encode the expert knowledge on how to classify the documents under the given categories [3]. Since there is a requirement of human intervention in knowledge engineering, researchers in 90's have proposed many machine learning techniques to automatically manage the text documents [3]. The

DOI : $10.5121 /$ ijdkp.2012.2104 
advantages of a machine learning based approach are that the accuracy is comparable to that achieved by human experts and no intervention from either knowledge engineers or domain experts needed for the construction of a document management tool [4]. Many text mining methods like document retrieval, clustering, classification, routing, filtering are often used for effective management of text documents. However, in this paper we concentrate only on classification of text documents.

The task of text classification is to assign a boolean value to each pair $\left(d_{j}, k_{i}\right) \in D \times K$, where ' $\mathrm{D}$ ' is the domain of documents and ' $\mathrm{K}$ ' is a set of predefined categories. The task is to approximate the true function $\phi: D \times K \rightarrow\{1,0\}$ by means of a function $\hat{\phi}: D \times K \rightarrow\{1,0\}$ such that $\phi$ and $\hat{\phi}$ coincide as much as possible. The function $\hat{\phi}$ is called a classifier. A classifier can be built by training it systematically using a set of training documents $\mathrm{D}$, where all of the documents belonging to $\mathrm{D}$ are labeled according to $\mathrm{K}[4,5]$. Text classification presents many challenges and difficulties. Some of them are: high dimensionality (thousands of features), loss of correlation between adjacent terms and understanding the complex semantics of the terms in a document. Thus to tackle these problems a number of methods have been reported in the literature for effective text document classification. In this paper we propose a new datastructure called status matrix suitable for text classification. The proposed method attempts to preserve the sequence of the term appearance in the query document which in turn helps in improving the classification accuracy.

The paper is organized as follows. A brief literature survey and the limitations of the existing models are presented in section 2. The working principle of the proposed method is presented in section 3. Details of experimental settings and results are presented in section 4 . The paper is concluded in section 5 .

\section{RELATED WORK}

In automatic text classification, it has been proved that the term is the best unit for text representation and classification [6]. Though a text document expresses vast range of information, unfortunately, it lacks the imposed structure of traditional database. Therefore, unstructured data, particularly free running text data has to be transformed into a structured data. To do this, many preprocessing techniques are proposed in literature [7, 8]. After converting an unstructured data into a structured data, we need to have an effective document representation model to build an efficient classification system. Bag of Word (BoW) is one of the basic methods of representing a document. The BoW is used to form a vector representing a document using the frequency count of each term in the document. This method of document representation is called as a Vector Space Model (VSM) [9]. The major limitation of VSM is that the correlation and context of each term is lost which is very important in understanding a document. Jain and $\mathrm{Li}$ [1] used binary representation for given document. The major drawback of this model is that it results in a huge sparse matrix, which raises a problem of high dimensionality. Hotho et al., [10] proposed an ontology representation for a document to keep the semantic relationship between the terms in a document. This ontology model preserves the domain knowledge of a term present in a document. However, automatic ontology construction is a difficult task due to the lack of structured knowledge base. Cavanar., (1994) [11] used a sequence of symbols (byte, a character or a word) called N-Grams, that are extracted from a long string in a document. In a N-Gram scheme, it is very difficult to decide the number of grams to be considered for effective document representation. Another approach [12] uses multi-word terms as vector components to represent a document. But this method requires a sophisticated automatic term extraction algorithms to extract the terms automatically from a document. Wei et al., (2008) proposed an approach called Latent Semantic Indexing (LSI) [13] 
which preserves the representative features for a document. The LSI preserves the most representative features rather than discriminating features. Thus to overcome this problem, Locality Preserving Indexing (LPI) [14] was proposed for document representation. The LPI discovers the local semantic structure of a document. Unfortunately LPI is not efficient in time and memory [15]. Choudhary and Bhattacharyya (2002) [16] used Universal Networking Language (UNL) to represent a document. The UNL represents the document in the form of a graph with words as nodes and relation between them as links. This method requires the construction of a graph for every document and hence it is unwieldy to use for an application where large numbers of documents are present.

After giving an effective representation for a document, the task of text classification is to classify the documents to the predefined categories. In order to do so, many statistical and computational models have been developed based on Naïve Bayes classifier [17, 18], K-NN classifier [19, 20], Centroid Classifier [21], Decision Trees [22, 23], Rocchio classifier [24] Neural Networks [25], Support Vector Machines [3, 26].

Although many text document representation models are available in literature, frequency-based BoW model gives effective results in text classification task. Indeed, till date the best multiclass, multi-labeled categorization results for well known datasets are based on BoW representation [27]. Unfortunately, BoW representation scheme has its own limitations. Some of them are: high dimensionality of the representation, loss of correlation with adjacent words and loss of semantic relationship that exist among the terms in a document [28]. Also the main problem with the frequency based approach is that given a term, with lesser frequency of occurrence may be appropriate in describing a document, whereas, a term with the higher frequency may have a less importance. Unfortunately, frequency-based BoW methods do not take this into account [16]. Hence there is a need for developing a new scheme for document representation preserving the correlation among adjacent words. This motivated us to use a new datastrcuture called "Status Matrix" [29] which effectively represents a text document and thereby giving a better classification results.

\section{PROPOSED METHOD}

In this section, we propose a new method of representing documents based on preserving the sequence of term occurrence in a document. Subsequently, we present the corresponding classification model.

\subsection{Representation Stage}

Let there be $k$ number of classes each containing $n$ number of documents. A simple text processing algorithm is employed to extract the terms (words) present in each document. From the extracted set of words, stop words are removed. For each class say $C_{j}, j=1,2, \ldots, k$, set of all words present in the documents of that class is formed. From these set of words an inverted list structure is formed for each of the word by associating the labels of the class of the documents that contain that particular word. The list of class labels associated with a word may contain many class labels as it is not uncommon that the documents of different classes contain the same word. The words and their associated lists of class indices are recommended to be stored in the knowledge base to support classification of an unknown test document.

However, this representation requires a linear time searching, which is not acceptable in real pragmatic scenario. Thus in order to speed up classification and to make the representation scheme dynamic supporting addition and deletion of documents, we recommend to index the documents using an existing indexing data structure. To do this task, one may think of many indexing structures like multidimensional binary trees [30], G-Tree [31], KDB Tree [32] and BD Tree [33]. However, each structure has got its own limitations [34] with respect to handling 
the data and storage methods. Thus, in our work we make use of B-Tree structure as it is simple and less complex. Moreover, B-Tree is used because of its availability, its simplicity and less complexity in addition to its balanced nature.

The proposed B-tree based system can be easily extended towards dynamic databases, as it is very easy to include new documents. In addition, insertion of new documents is as simple as just the insertion of set of words into the existing set and updating the associated term lists. With respect to the proposed representation scheme, insertion of a document into the database is simply a process of inserting the terms present in the document into the B-tree. In order to insert a term $T$ corresponding to the document to be inserted, the B-tree is accessed through to find out the location of $T$ in the B-tree. If $T$ is already present in the database, the insertion problem is reduced to the problem of getting the list of documents updated by appending the index of the document to be inserted. On the other hand, if a term $T$ is not present in the database then no doubt we are at the node $U$ where $T$ is expected to be present. If $U$ contains fewer than $(r-1)$ terms ( $r$ is the order of the B-tree), $T$ is simply inserted into $U$ in a sorted order. Otherwise, unlike conventional B-tree insertion procedure where the node is eventually split into two nodes, in our work, we recommend to look at the siblings of the node to find if any a free location, so that by data movements we can get the $T$ term accommodated at the node $U$ itself without splitting it up. Indeed this modification suggested to the conventional Btree insertion process significantly enhances the efficacy of the insertion procedure particularly on a very large B-tree. The complexity of using the B-tree is of $O\left(\log _{r} t\right)$, where $t$ is the number terms stored in the B-tree and $r$ is the order of the B-tree.

For an illustrative purpose, we consider four different classes of documents. For each class we have created a knowledge base as follows. Given a set of training documents of an individual class, stopwords from each training documents are eliminated and the terms are pooled to form a knowledge base. The knowledge base obtained for four different classes are given below:

K1: categorization, documents,implement,metric,similarity,text

K2: algorithms,categorization,mining,similarity,video

K3: algorithms,efficient,enhancements,filter,image

K4: algorithms, congestion, networks, protocols,routing

The terms present in the knowledge base along with their class labels are stored in a B-tree for the purpose of fast retrieval.

A B-tree of order $r=3$ is constructed (Figure 1) to store the distinct terms and each term in the B-tree is attached with its respective list of class indices. The index table containing all terms for each of the documents to be stored is created as shown in Table 1.

\subsection{Classification}

Sequence of occurrence of words in any text plays a major role in understanding the text document. However, most of the existing methods do not preserve the sequence of occurrence of words as they assume that the word occurrence is independent of text representation.

Simple method to check the sequence of occurrence of words is same as common longest substring matching. Thus, one can think that the problem of classifying a test document is reduced to the problem of finding out a common longest subsequence of terms in the database. In practice, this is not acceptable as the process of substring matching has non-deterministic polynomial time complexity. 
Hence, in this section we propose an alternative method of matching and classification of text documents. For the purpose of preserving the sequence of occurrences of words in a test document we recommend to use the concept of status matrix. Status matrix representation was proposed for the purpose of recognition of partially occluded object recognition, where status matrix representation is a binary matrix preserving the order in which the information occurs.

\subsection{Computational Complexity of Classification}

As there are $k$ classes and a query document contains $t_{q}$ terms, we require $O\left(t_{q} \log _{r} t\right)$ computations to create a status matrix of size $M$.

A status matrix is a binary matrix where the entries are either 0 or 1 . The status matrix is of dimension $k \times t_{q}$ where, $k$ is the number of classes, and $t_{q}$ is the number of terms in the query text document after preprocessing.

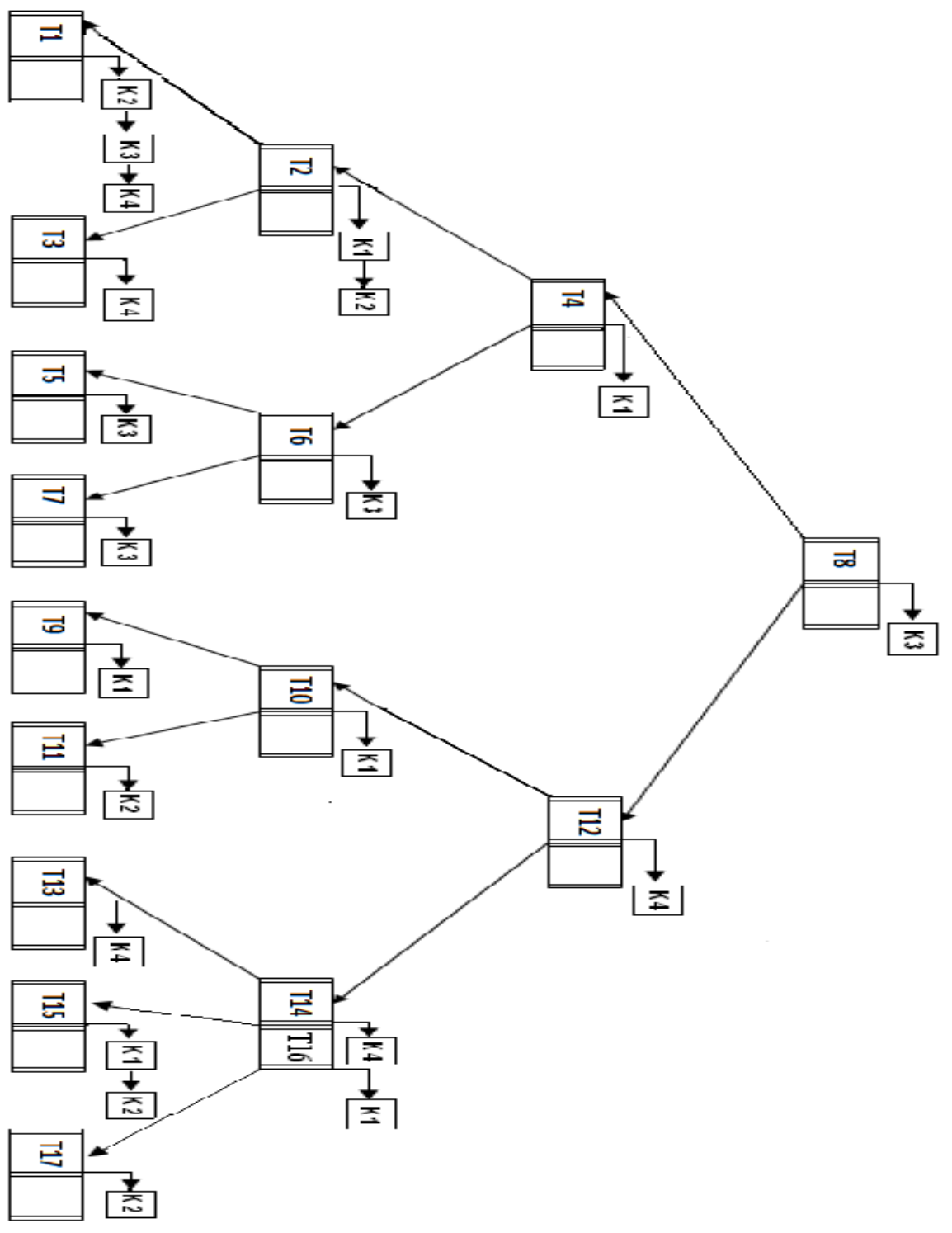


Figure 1: A B-tree representation to the knowledge base

Table 1. The index table for the illustrated example

\begin{tabular}{|l|l|l|l|}
\hline Index & Terms & Index & Terms \\
\hline T1 & Algorithms & T 10 & Metric \\
\hline T2 & Categorization & T 11 & Mining \\
\hline T3 & Congestion & T 12 & Networks \\
\hline T 4 & Documents & T 13 & Protocols \\
\hline T 5 & Efficient & T 14 & Routing \\
\hline T 6 & Enhancements & T 15 & Similarity \\
\hline T 7 & Filter & T 16 & Text \\
\hline T 8 & Image & T 17 & Video \\
\hline T 9 & Implement & & \\
\hline
\end{tabular}

The B-tree is accessed through in search of each term and the lists of document indices corresponding to that term are retrieved from the database. If the $i^{\text {th }}$ term $T_{i}$ of the query document is present in the knowledge base of the class $C_{j}$, then the entry corresponding to the row of $C_{j}$ and the column $T_{i}$ in the status matrix is set to 1 , otherwise it is set to 0 . That is, if $M$ is a status matrix, then, $M$ is given by

$$
M_{i j}= \begin{cases}1 & \text { if } T_{i} \in C_{j} \\ 0 & \text { otherwise }\end{cases}
$$

Assuming each row of the status matrix as a binary string, we then look for a row with a longest substring containing only 1s. The class corresponding to that row is declared to be the class of the test document. As an illustration, let us consider the following paragraph as a query document $d_{q}$.

"Text categorization is not a trivial problem. The complexity of the problem lies in how to define a similarity metric between the documents, and then how to implement a computationally efficient algorithm to solve the problem given this similarity metric".

In order to classify this document we first eliminate stopwords present in it, which results with the following set of terms.

\{text, categorization, trivial, problem, complexity, similarity, metric, documents, implement, computationally, efficient, algorithms, similarity, metric\}.

This query document totally contains 14 terms. Now it is understood that as there are 4 classes and the query document has 14 terms, we have the status matrix of size $4 \times 14$ as shown in Table 2 .

Once the status matrix is constructed we search through the status matrix in search of longest matched sequence. Now, the test document is assigned to the class which has longest matched sequence of terms present in the query document. Here in this example the query document is given the class label 1.

Table 2. Status matrix obtained for query document $d_{q}$

\begin{tabular}{|l|l|l|l|l|l|l|l|l|l|l|l|l|l|l|}
\hline & $\mathrm{T} 1$ & $\mathrm{~T}_{2}$ & $\mathrm{~T}_{3}$ & $\mathrm{~T}_{4}$ & $\mathrm{~T}_{5}$ & $\mathrm{~T}_{6}$ & $\mathrm{~T}_{7}$ & $\mathrm{~T}_{8}$ & $\mathrm{~T}_{9}$ & $\mathrm{~T}_{10}$ & $\mathrm{~T}_{11}$ & $\mathrm{~T}_{12}$ & $\mathrm{~T}_{13}$ & $\mathrm{~T}_{14}$ \\
\hline $\mathrm{k}_{1}$ & 1 & 1 & 0 & 0 & 0 & $\mathbf{1}$ & $\mathbf{1}$ & $\mathbf{1}$ & $\mathbf{1}$ & 0 & 0 & 0 & 1 & 1 \\
\hline $\mathrm{k}_{2}$ & 0 & 1 & 0 & 0 & 0 & 1 & 0 & 0 & 0 & 0 & 0 & 1 & 1 & 0 \\
\hline
\end{tabular}


International Journal of Data Mining \& Knowledge Management Process (IJDKP) Vol.2, No.1, January 2012

\begin{tabular}{|l|l|l|l|l|l|l|l|l|l|l|l|l|l|l|}
\hline $\mathrm{k}_{3}$ & 0 & 0 & 0 & 0 & 0 & 0 & 0 & 0 & 0 & 0 & 1 & 1 & 0 & 0 \\
\hline $\mathrm{k}_{4}$ & 0 & 0 & 0 & 0 & 0 & 0 & 0 & 0 & 0 & 0 & 0 & 1 & 0 & 0 \\
\hline
\end{tabular}

\section{EXPERIMENTAL SETUP}

\subsection{Datasets}

To test the efficacy of the proposed model, we have used the following five datasets. The first dataset is standard 20 Newsgroup Large [35]. It contains 20000 documents categorized into 20 classes. The second dataset consists of vehicle characteristics extracted from wikipedia pages (vehicles- wikipedia) [2]. The dataset contains 4 categories that have low degrees of similarity. The dataset contains four categories of vehicles: Aircraft, Boats, Cars and Trains. All the four categories are easily differentiated and every category has a set of unique key words. The third dataset is a standard 20 mini newsgroup dataset which contains about 2000 documents evenly divided among 20 Usenet discussion groups. This dataset is a subset of 20 newsgroups which contains 20,000 documents. In 20 MiniNewsgroup, each class contains 100 documents in 20 classes which are randomly picked from original dataset. The fourth dataset is constructed by a text corpus of 1000 documents that are downloaded from Google-Newsgroup. Each class contains 100 documents belonging to 10 different classes (Business, Cricket, Music, Electronics, Biofuels, Biometrics, Astronomy, Health, Video Processing and Text Mining). The fifth dataset is a collection of research article abstracts. All these research articles are downloaded from the scientific web portals. We have collected 1000 documents from 10 different classes. Each class contains 100 documents.

\subsection{Experimentation}

In this section, we present the results of the experiments conducted to demonstrate the effectiveness of the proposed status matrix based method on all the five datasets viz., 20 newsgroup large, vehicles wikipedia, 20 mini newsgroup, google newsgroup and research article abstracts. During experimentation, we conducted two different sets of experiments. In the first set of experiments, we used, $50 \%$ of the documents of each class of a dataset to create training set and the remaining $50 \%$ of the documents for testing purpose. On the other hand, in the second set of experiments, the numbers of training and testing documents are in the ratio 60:40. Both experiments are repeated 5 times by choosing the training samples randomly. As measures of goodness of the proposed method, we computed both accuracy and F measure. The minimum, maximum and the average value of the classification accuracy of all the 5 trials are presented in Table 3. Similarly, Table 4 presents the minimum, maximum and the average value of the F Measure of all the 5 trials.

Figure 2 shows classwise maximum accuracy values obtained among all 5 trials for all the five datasets by the proposed status matrix based method in experiment 1 (50:50 case). Figure 3 shows the average accuracy values of all the 5 trials for all the five datasets obtained by the proposed status matrix method based in experiment 1 (50:50). Figure 4 shows classwise maximum accuracy values obtained among all 5 trials for all the five datasets by the proposed status matrix based method in experiment 2 (60:40). Figure 5 shows the average accuracy values of all the 5 trials for all the five datasets obtained by the proposed status matrix based method in experiment $2(60: 40)$.

Figure 6 shows classwise maximum $\mathrm{F}$ Measure values obtained among all 5 trials for all the five datasets by the proposed status matrix based method in experiment 1 (50:50 case). Figure 7 shows the average $\mathrm{F}$ Measure values of all the 5 trials for all the five datasets obtained by the proposed status matrix based method in experiment 1 (50:50). Figure 8 shows classwise maximum $\mathrm{F}$ Measure values obtained among all 5 trials for all the five datasets by the status matrix based method in experiment 2 (60:40). Figure 9 shows the average $F$ Measure values of 
all the 5 trials for all the five datasets obtained by the proposed status matrix based method in experiment $2(60: 40)$.

It is observed that the proposed status matrix based method has better accuracy and F Measure in experiment 2 when compared to that of experiment 1.

Table 5 shows the comparative analysis of the results on five datasets mentioned above. It is clear from the Table 5, that the proposed model achieved good classification accuracy on three benchmark datasets and also on our own dataset (Google Newsgroup and Research article abstracts), when compared with other well known classifiers viz. Naïve Bayes, KNN and SVM classifier. This is because of the proposed model has a capability of classifying the documents at two stages (one is at voting stage and the other is at term sequence stage). It is also worth mentioning that the incorporation of status matrix improves the performance of a voting classifier.

\section{CONCLUSION}

In this paper, we have proposed the classification of text documents using B-Tree. Further, we have presented a new datastructure called status matrix through which, we make an attempt to preserve the sequence of term occurrence in the query document. In addition, in order to avoid sequential matching during classification, we propose to index the terms in B-tree, an efficient index scheme. In order to investigate the effectiveness and robustness of the proposed method, experimentation is conducted on five different datasets. The experimental results tabulated in Table 4, indicate that the proposed method offers better performance results than other three well-known classifiers. In the proposed method we have pooled the terms of training documents of each class to create a knowledge base. For a given query document we create the status matrix to preserve the sequence of the term appearance in the query document. As we have pooled the terms in the knowledge base we are not preserving the term sequence during training stage. Along with this the presence of continuous 1's in status matrix do not ensure that the database contains any document having same sequence of terms present in the test document. Hence in our future work we try to study the sequence of the term appearance using the concept of status matrix even on training documents and there by preserving the topological sequence of term occurrence in a document useful for semantic retrieval.

Table 3. Classification accuracy of the proposed status matrix based method on different data sets

\begin{tabular}{|c|c|c|c|c|}
\hline \multirow{2}{*}{ Dataset } & $\begin{array}{c}\text { Training } \\
\text { Vs } \\
\text { Testing }\end{array}$ & $\begin{array}{c}\text { Minimum } \\
\text { Accuracy } \\
\text { (5 Trials) }\end{array}$ & $\begin{array}{c}\text { Maximum } \\
\text { Accuracy } \\
\text { (5 Trials) }\end{array}$ & $\begin{array}{c}\text { Average } \\
\text { Accuracy } \\
\text { (5 Trials) }\end{array}$ \\
\hline $\begin{array}{c}\text { 20 Newsgroup } \\
\text { Large }\end{array}$ & 50 vs 50 & 79.65 & 84.20 & 82.64 \\
\cline { 2 - 5 } & 60 vs 40 & 84.35 & 87.85 & 86.35 \\
\hline \multirow{2}{*}{$\begin{array}{c}\text { Vehicles } \\
\text { Wikipedia }\end{array}$} & 50 vs 50 & 70.65 & 72.85 & 71.60 \\
\cline { 2 - 5 } & 60 vs 40 & 74.95 & 76.00 & 75.45 \\
\hline $\begin{array}{c}20 \text { Mini } \\
\text { Newsgroup }\end{array}$ & 50 vs 50 & 64.65 & 68.95 & 66.91 \\
\cline { 2 - 5 } & 60 vs 40 & 71.00 & 76.85 & 73.95 \\
\hline $\begin{array}{c}\text { Google } \\
\text { Newsgroup }\end{array}$ & 50 vs 50 & 86.70 & 89.70 & 88.74 \\
\cline { 2 - 5 } Research & 60 vs 40 & 89.85 & 96.00 & 93.33 \\
\cline { 2 - 5 } $\begin{array}{c}\text { Article } \\
\text { Abstracts }\end{array}$ & 50 vs 50 & 86.25 & 90.25 & 88.52 \\
\cline { 2 - 5 } & 60 vs 40 & 89.00 & 91.25 & 90.13 \\
\hline
\end{tabular}


Table 4. F measure values of the proposed status matrix based method on different data sets

\begin{tabular}{|c|c|c|c|c|}
\hline \multirow{2}{*}{ Dataset } & $\begin{array}{c}\text { Training } \\
\text { Vs } \\
\text { Testing }\end{array}$ & $\begin{array}{c}\text { Minimum } \\
\text { F Measure } \\
\text { (5 Trials) }\end{array}$ & $\begin{array}{c}\text { Maximum } \\
\text { F Measure } \\
\text { (5 Trials) }\end{array}$ & $\begin{array}{c}\text { Average } \\
\text { F Measure } \\
\text { (5 Trials) }\end{array}$ \\
\hline $\begin{array}{c}\text { 20 Newsgroup } \\
\text { Large }\end{array}$ & 50 vs 50 & 0.820 & 0.849 & 0.835 \\
\cline { 2 - 5 } & 60 vs 40 & 0.880 & 0.891 & 0.886 \\
\hline $\begin{array}{c}\text { Vehicles } \\
\text { Wikipedia }\end{array}$ & 50 vs 50 & 0.827 & 0.854 & 0.842 \\
\cline { 2 - 5 } & 60 vs 40 & 0.885 & 0.905 & 0.892 \\
\hline $\begin{array}{c}20 \text { Mini } \\
\text { Newsgroup }\end{array}$ & 50 vs 50 & 0.867 & 0.882 & 0.876 \\
\cline { 2 - 5 } & 60 vs 40 & 0.865 & 0.895 & 0.883 \\
\hline $\begin{array}{c}\text { Google } \\
\text { Newsgroup }\end{array}$ & 50 vs 50 & 0.875 & 0.890 & 0.883 \\
\cline { 2 - 5 } & 60 vs 40 & 0.880 & 0.920 & 0.904 \\
\hline $\begin{array}{c}\text { Research } \\
\text { Article } \\
\text { Abstracts }\end{array}$ & 50 vs 50 & 0.862 & 0.907 & 0.890 \\
\cline { 2 - 5 } & 60 vs 40 & 0.918 & 0.926 & 0.921 \\
\hline
\end{tabular}

Table 5. Comparative analysis of the proposed method with other classifiers.

\begin{tabular}{|c|c|c|c|c|c|}
\hline Dataset Used & $\begin{array}{c}\text { Voting } \\
\text { Classifier }\end{array}$ & $\begin{array}{c}\text { Voting + } \\
\text { Status } \\
\text { Matrix } \\
\text { Classifier } \\
\text { (Proposed } \\
\text { Method) }\end{array}$ & $\begin{array}{c}\text { Naïve } \\
\text { Bayes } \\
\text { Classifier }\end{array}$ & $\begin{array}{c}\text { KNN } \\
\text { Classifier }\end{array}$ & $\begin{array}{c}\text { SVM } \\
\text { Classifier }\end{array}$ \\
\hline $\begin{array}{c}\text { 20 Newsgroup } \\
\text { Large }\end{array}$ & 82.55 & 87.85 & 86.50 & 70.00 & 85.65 \\
\hline $\begin{array}{c}\text { Google } \\
\text { Newsgroup }\end{array}$ & 93.50 & 96.00 & 80.00 & 46.25 & 48.25 \\
\hline Vehicle-Wiki & 67.50 & 76.00 & 74.00 & 64.50 & 63.00 \\
\hline $\begin{array}{c}\text { 20 Mini } \\
\text { Newsgroup }\end{array}$ & 66.25 & 71.12 & 66.22 & 38.73 & 51.02 \\
\hline $\begin{array}{c}\text { Research } \\
\text { Article } \\
\text { Abstracts }\end{array}$ & 86.75 & 91.25 & - & - & - \\
\hline
\end{tabular}




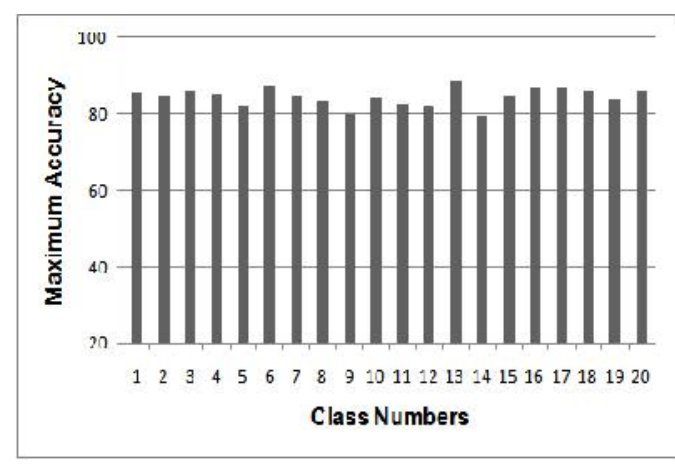

(a)

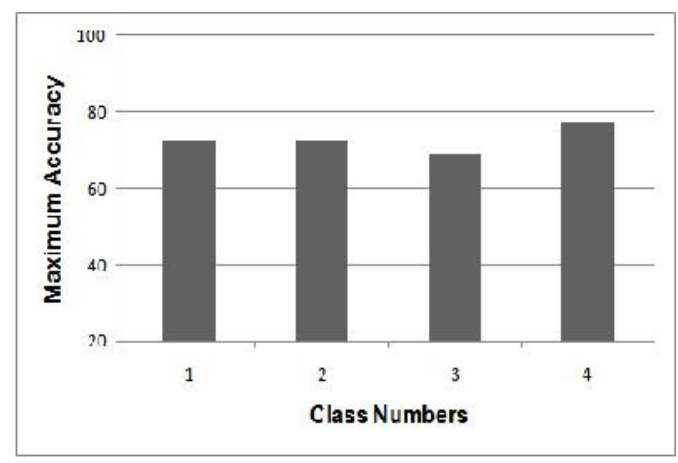

(c)

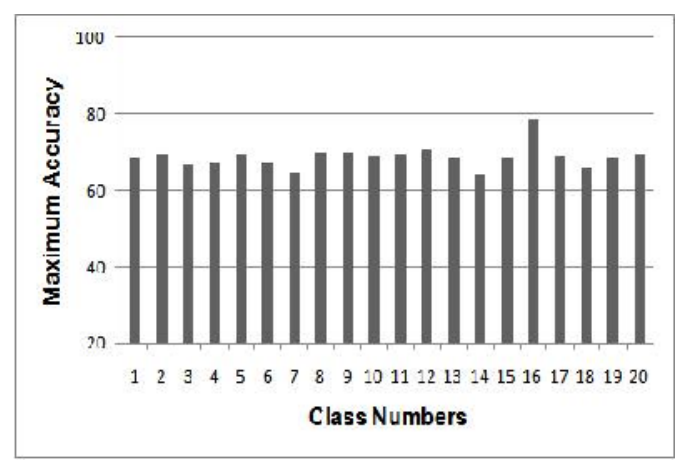

(b)

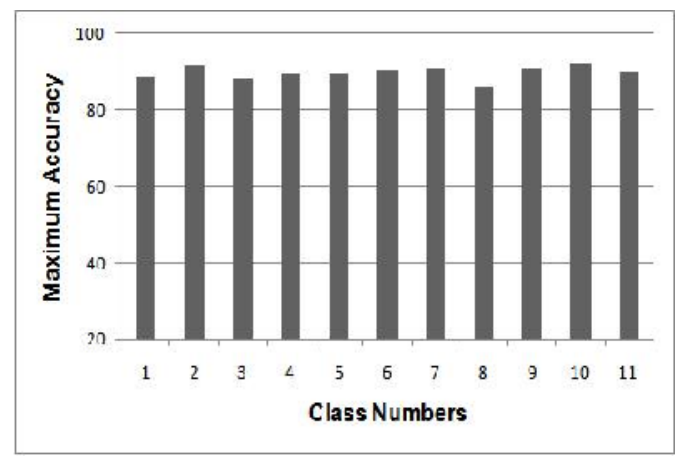

(d)

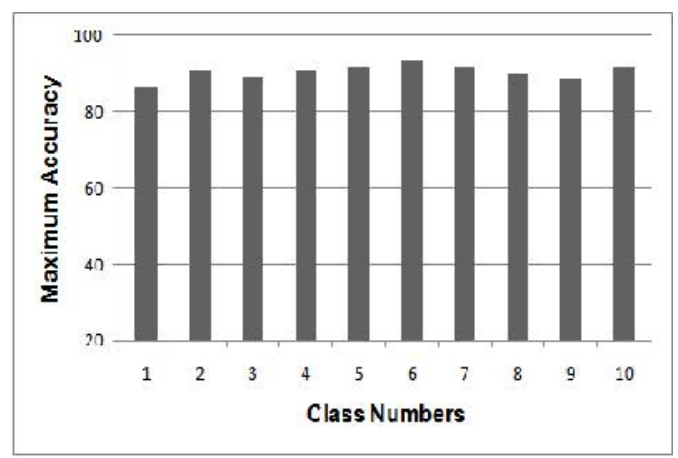

(e)

Figure 2. Classwise maximum accuracy values obtained among all 5 trials for all the five datasets by the proposed status matrix based method in experiment 1 (50:50)

(a) 20 Newsgroup Large, (b) 20 Mini Newsgroup, (c) Vehicles Wikipedia, (d) Google Newsgroup, (e) Research Article Abstracts 


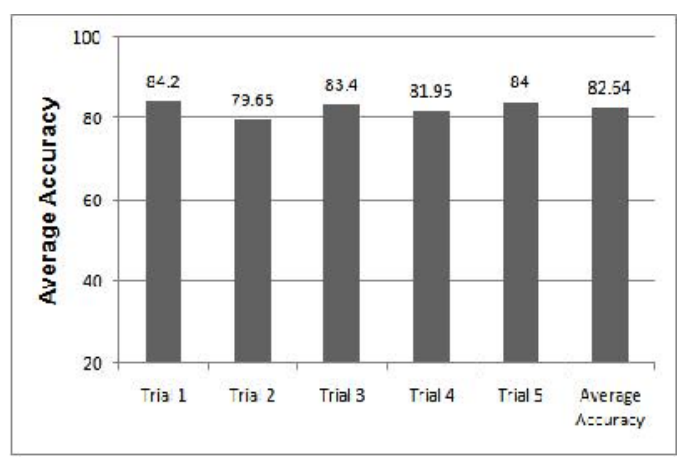

(a)

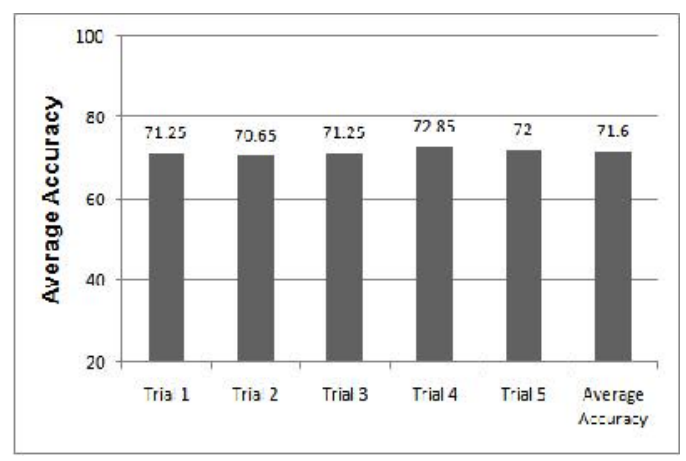

(c)

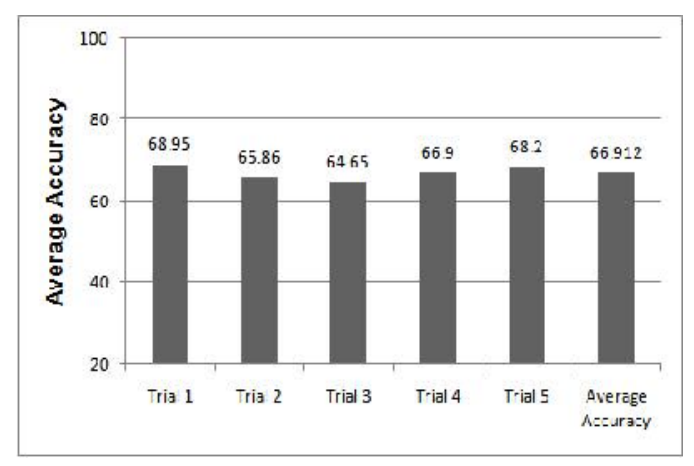

(b)

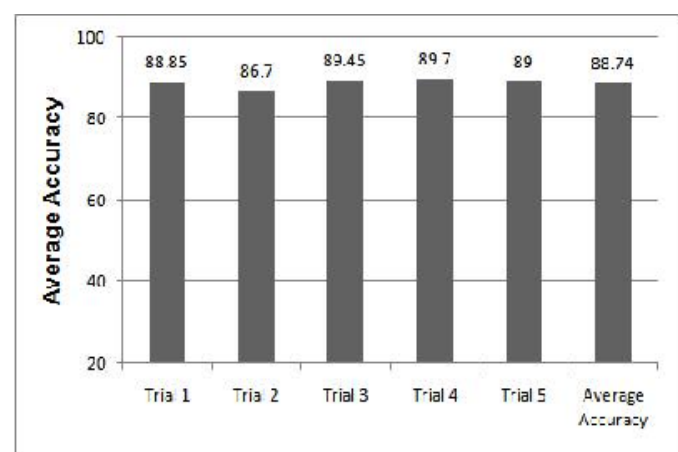

(d)

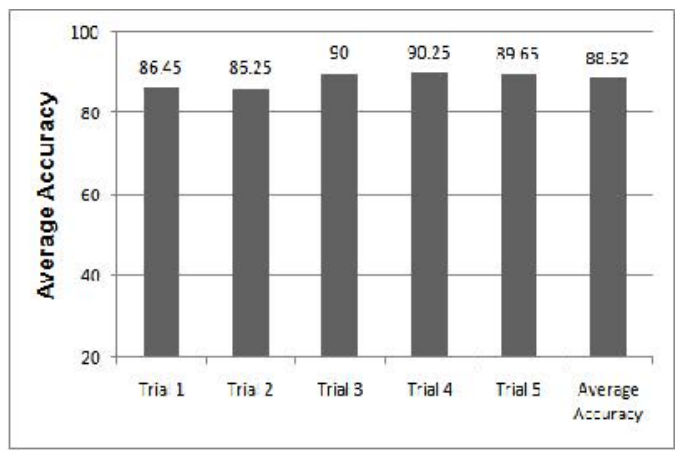

(e)

Figure 3. Average accuracy values of all 5 trials for all the five datasets obtained by the proposed status matrix based method in experiment 1 (50:50)

(a) 20 Newsgroup Large, (b) 20 Mini Newsgroup, (c) Vehicles Wikipedia, (d) Google Newsgroup, (e) Research Article Abstracts 
International Journal of Data Mining \& Knowledge Management Process (IJDKP) Vol.2, No.1, January 2012

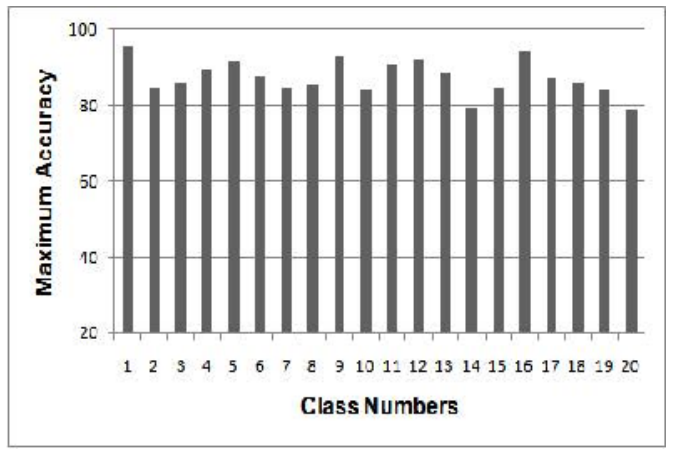

(a)

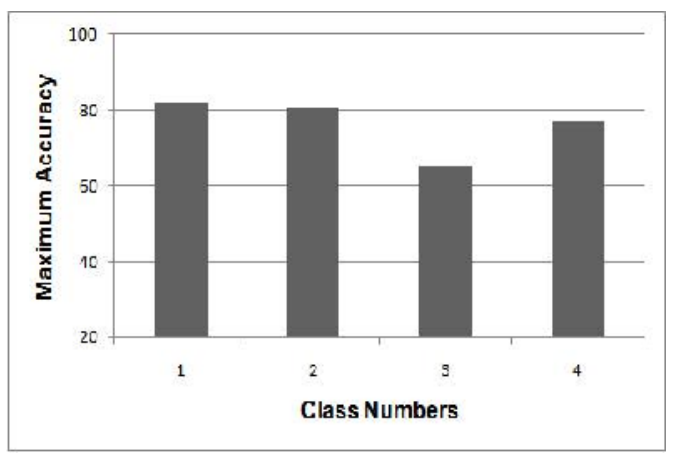

(c)

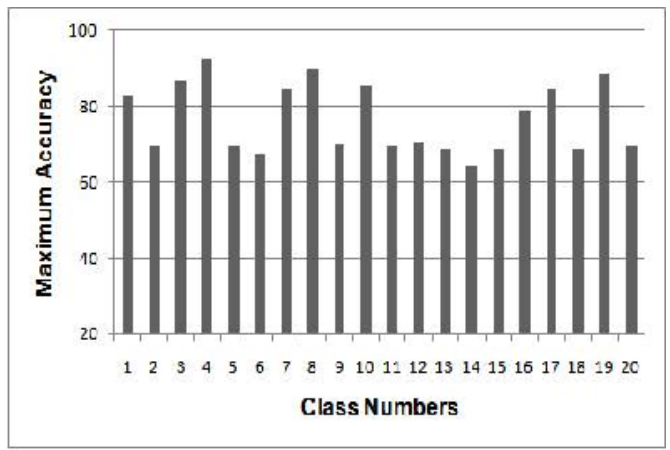

(b)

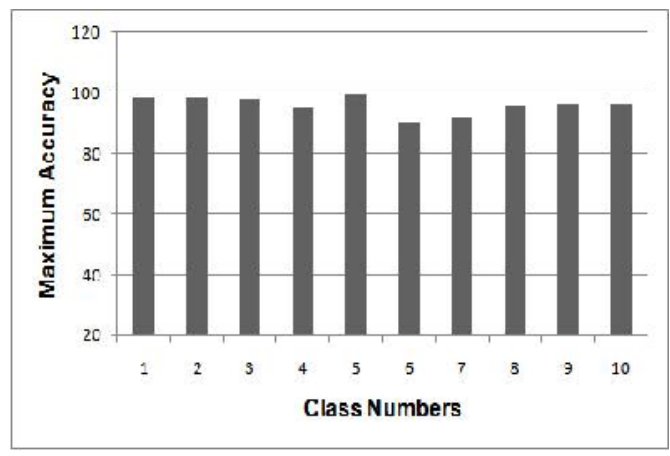

(d)

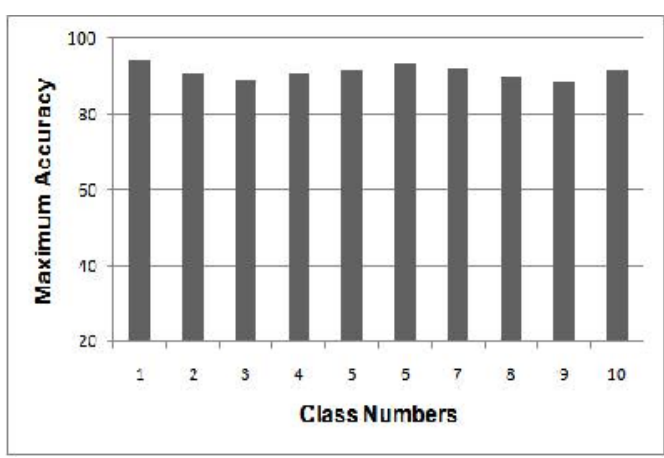

(e)

Figure 4. Classwise maximum accuracy values obtained among all 5 trials for all the five datasets by the proposed status matrix based method in experiment 2 (60:40)

(a) 20 Newsgroup Large, (b) 20 Mini Newsgroup, (c) Vehicles Wikipedia, (d) Google Newsgroup, (e) Research Article Abstracts 


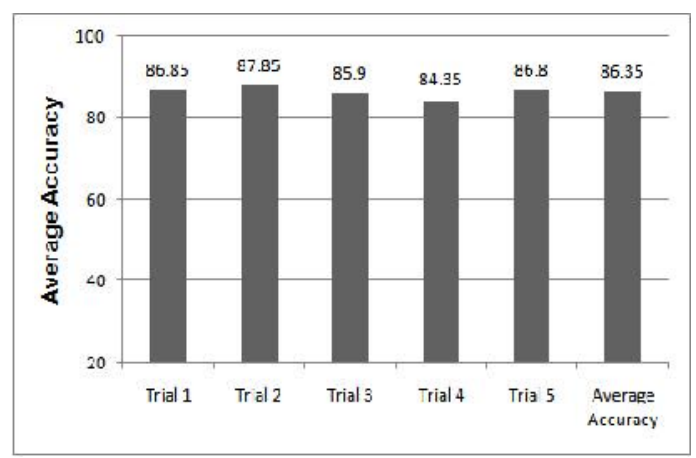

(a)

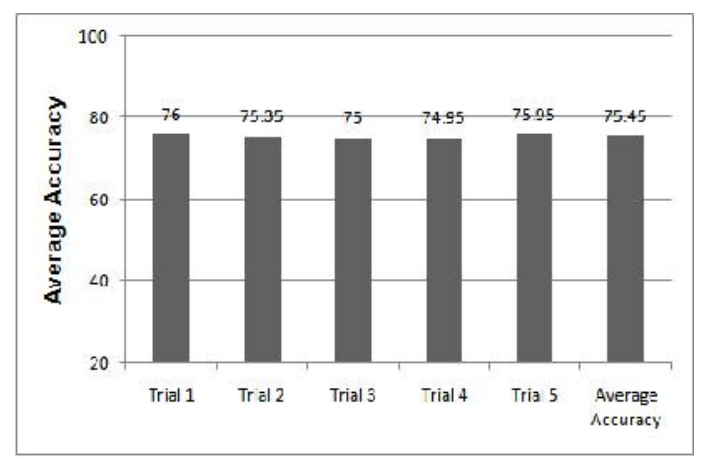

(c)

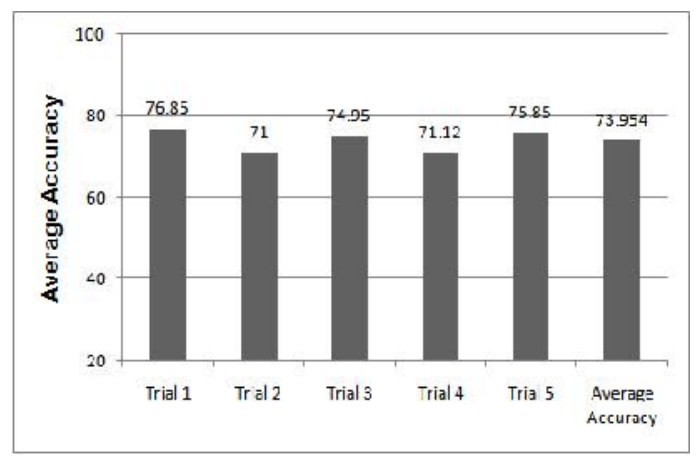

(b)

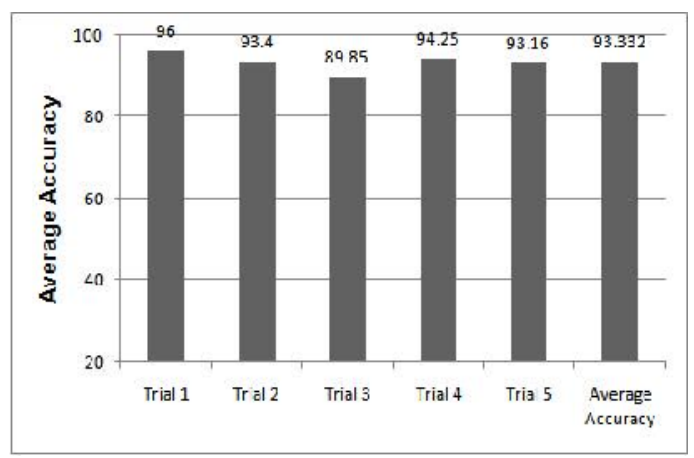

(d)

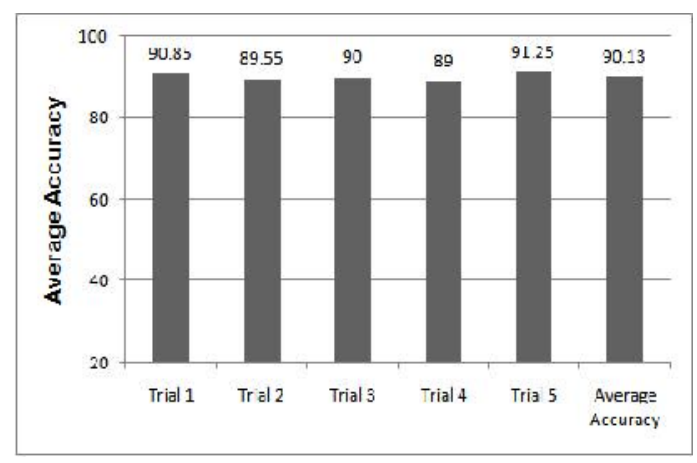

(e)

Figure 5. Average accuracy values of all 5 trials for all the five datasets obtained by the proposed status matrix based method in experiment 2 (60:40)

(a) 20 Newsgroup Large, (b) 20 Mini Newsgroup, (c) Vehicles Wikipedia, (d) Google Newsgroup, (e) Research Article Abstracts 


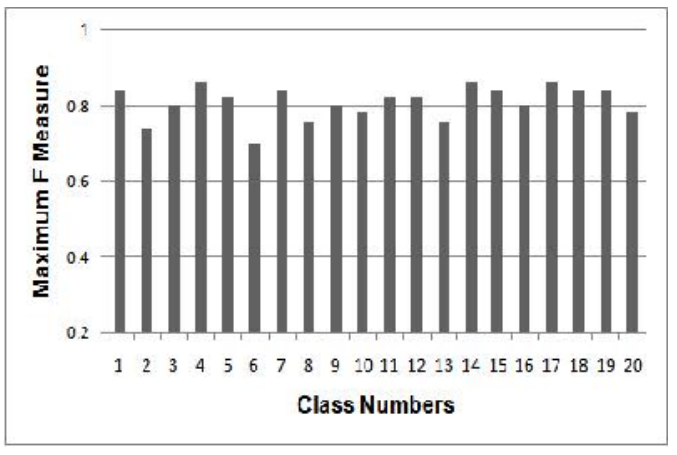

(a)

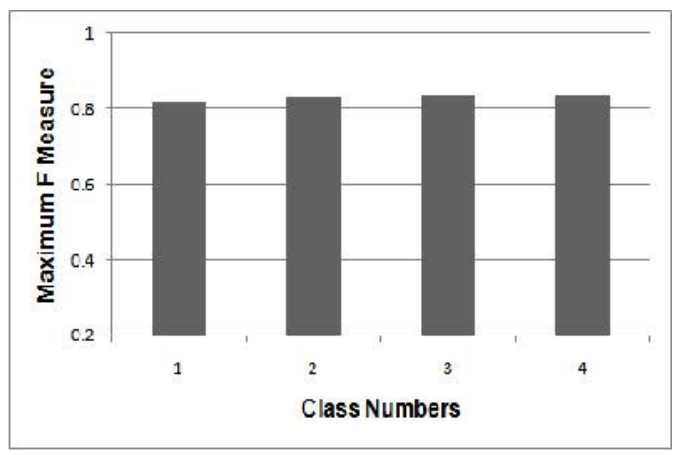

(c)

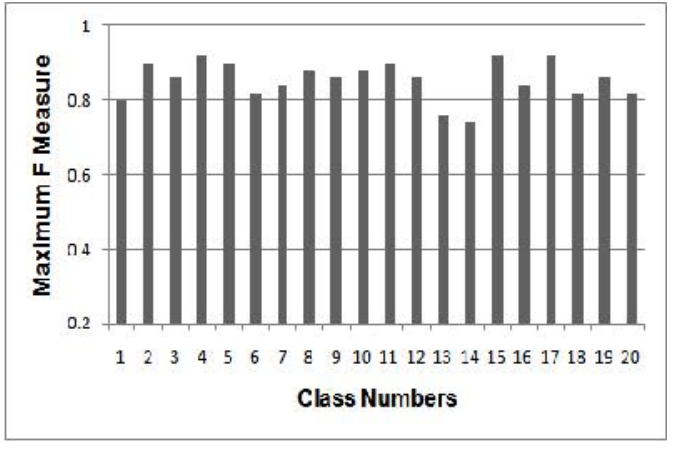

(b)

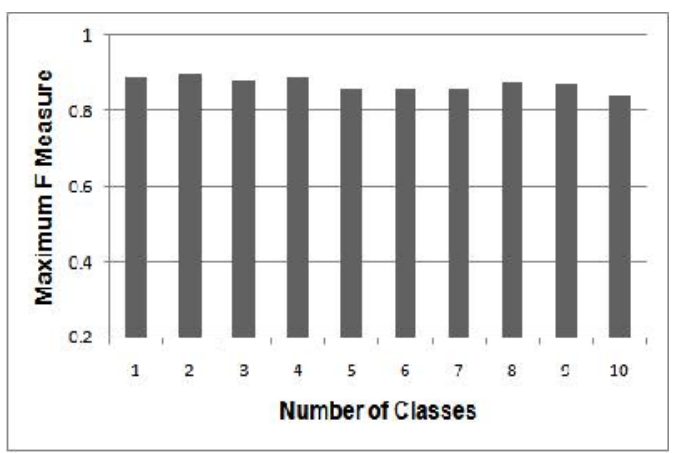

(d)

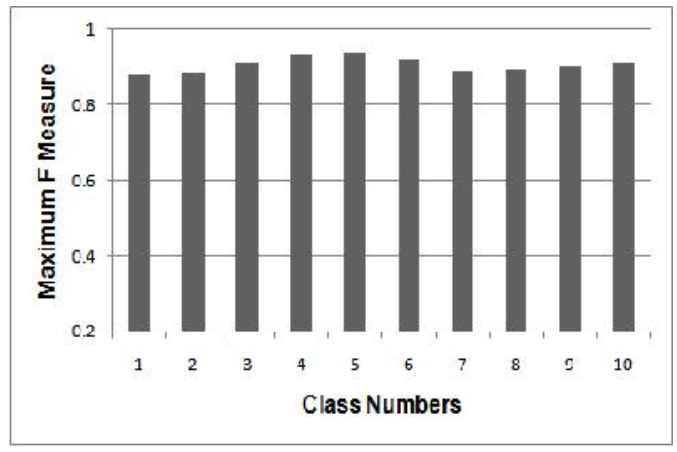

(e)

Figure 6. Classwise maximum F measure values obtained among all 5 trials for all the five datasets by the proposed status matrix based method in experiment 1 (50:50)

(a) 20 Newsgroup Large, (b) 20 Mini Newsgroup, (c) Vehicles Wikipedia, (d) Google Newsgroup, (e) Research Article Abstracts 


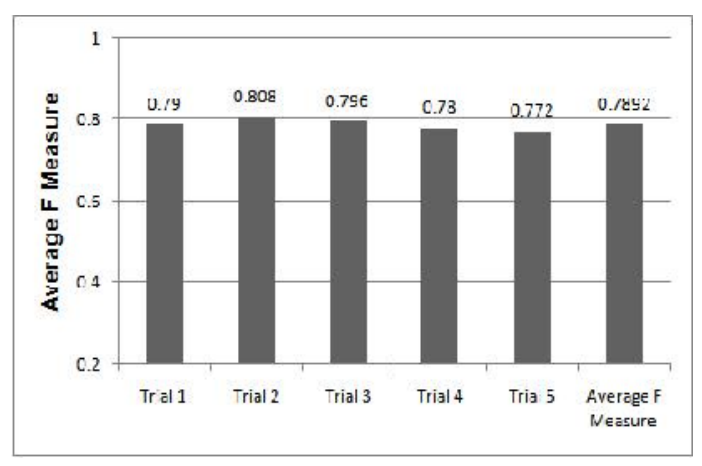

(a)

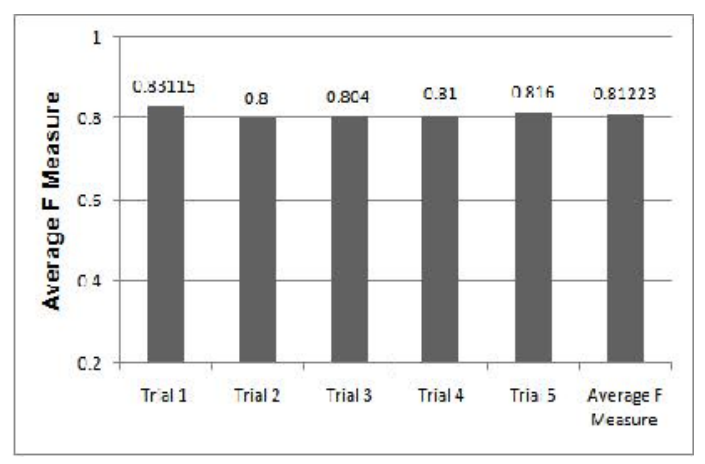

(c)

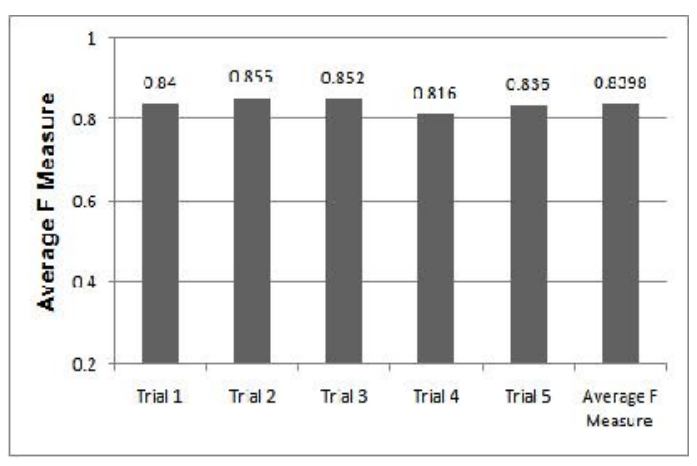

(b)

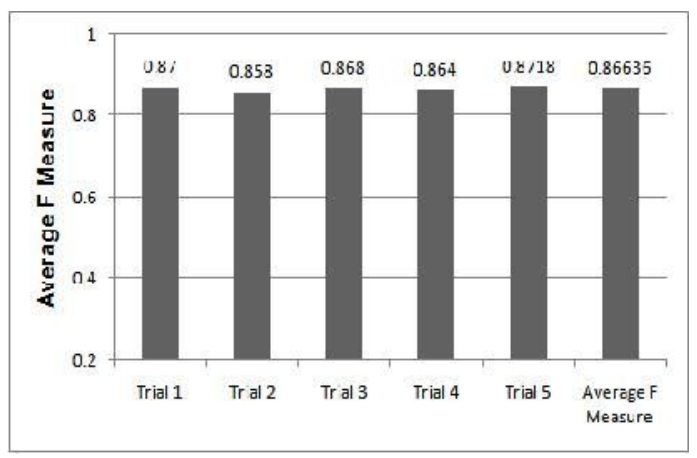

(d)

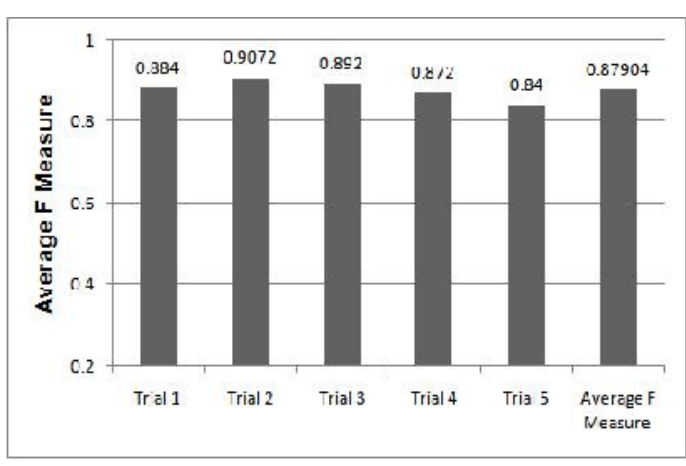

(e)

Figure 7. Average F measure values of all 5 trials for all the five datasets obtained by the proposed proposed status matrix based method in experiment 1 (50:50)

(a) 20 Newsgroup Large, (b) 20 Mini Newsgroup, (c) Vehicles Wikipedia, (d) Google Newsgroup, (e) Research Article Abstracts 


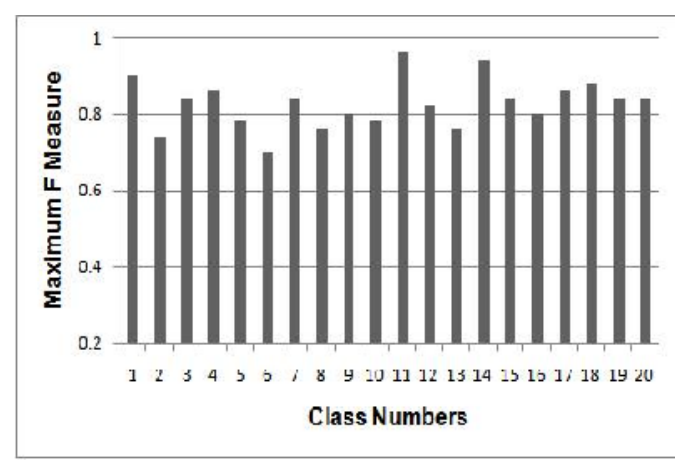

(a)

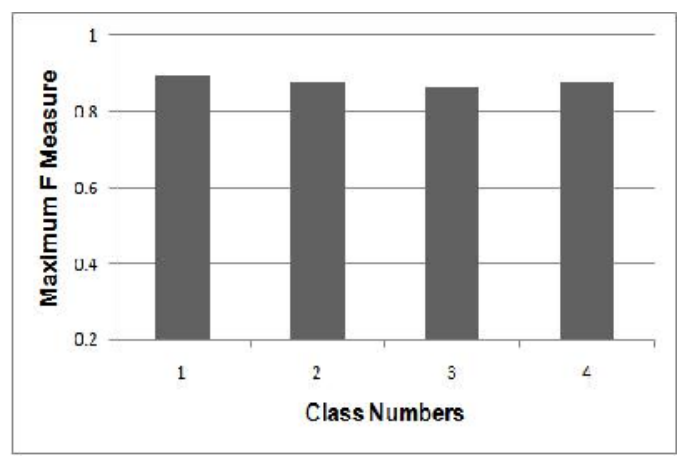

(c)

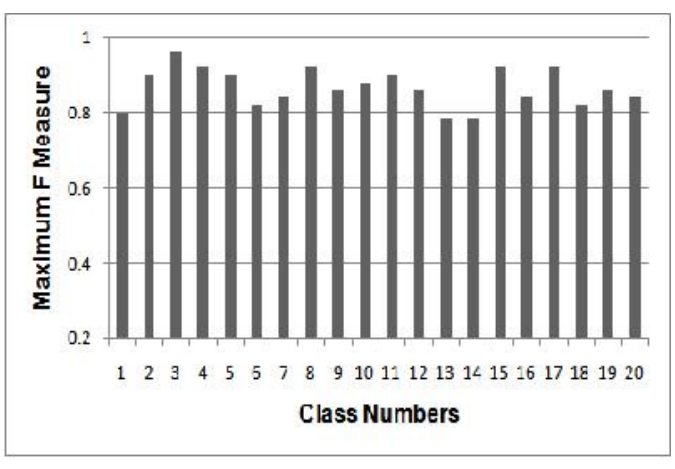

(b)

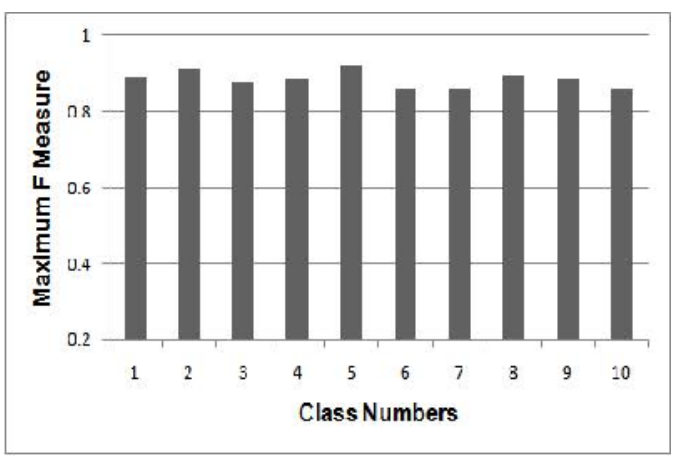

(d)

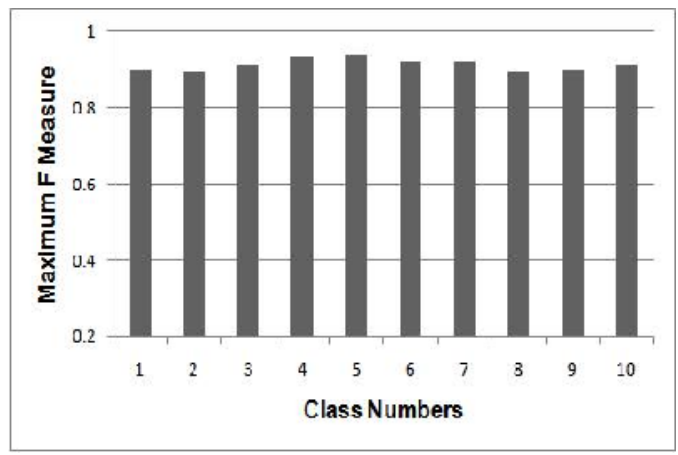

(e)

Figure 8. Classwise maximum F measure values obtained among all 5 trials for all the five datasets by the proposed status matrix based method in experiment $2(60: 40)$

(a) 20 Newsgroup Large, (b) 20 Mini Newsgroup, (c) Vehicles Wikipedia, (d) Google Newsgroup, (e) Research Article Abstracts 


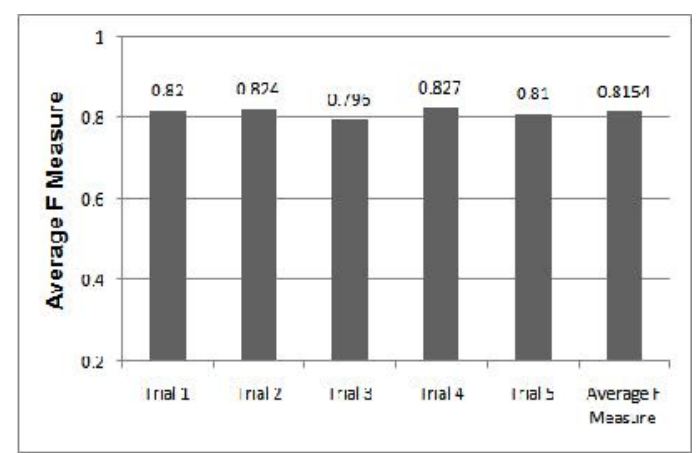

(a)

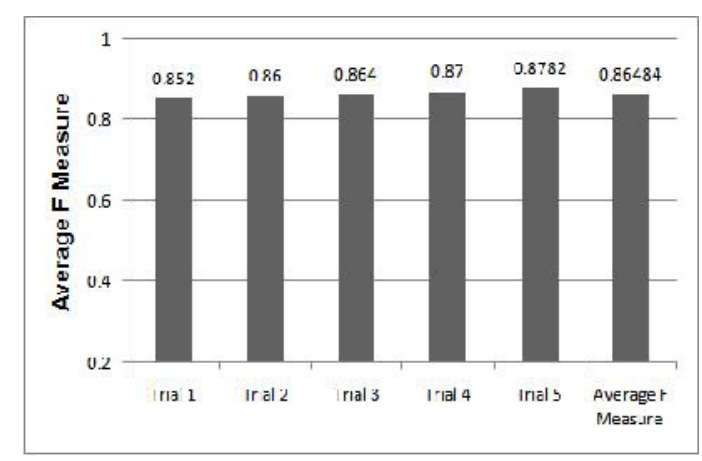

(c)

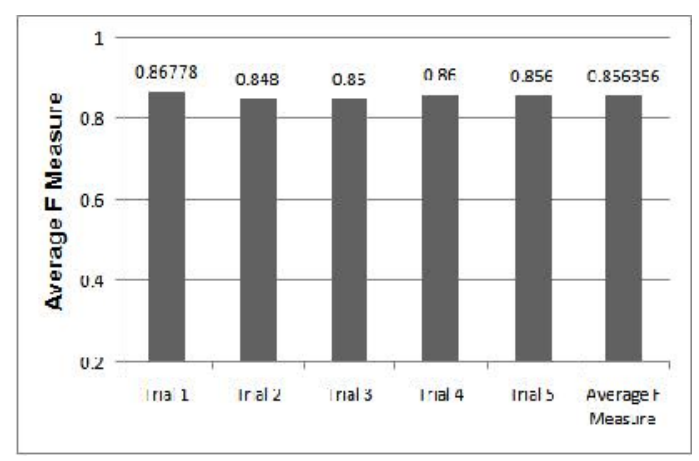

(b)

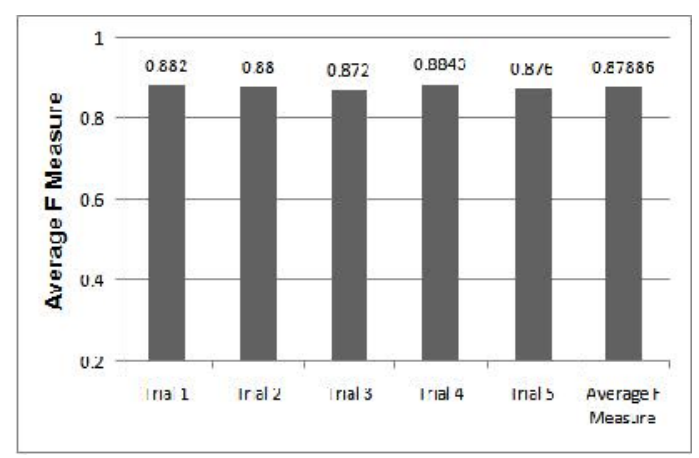

(d)

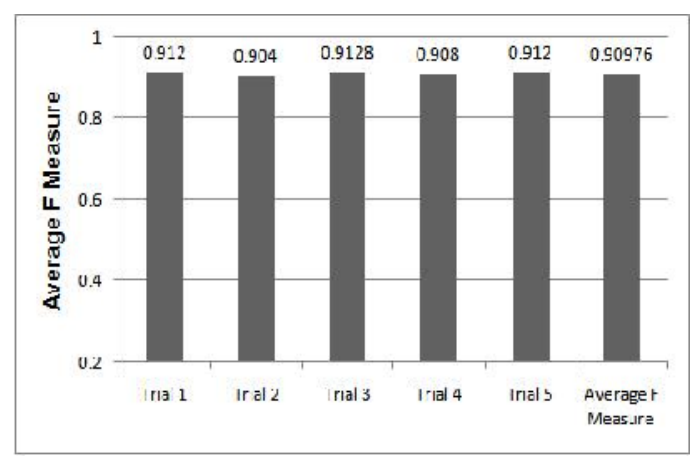

(e)

Figure 9. Average F measure values of all 5 trials for all the five datasets obtained by the proposed status matrix based method in experiment 2 (60:40)

(a) 20 Newsgroup Large, (b) 20 Mini Newsgroup, (c) Vehicles Wikipedia, (d) Google Newsgroup, (e) Research Article Abstracts 


\section{REFERENCES}

[1]. Li, Y.H., Jain, A.K.: Classification of Text Documents. The Computer Journal. vol. 41, pp. 537--546 (1998)

[2]. Isa, D. Lee, L.H., Kallimani, V.P., Rajkumar, R.: Text document preprocessing with the Bayes formula for classification using the support vector machine. IEEE Transactions on Knowledge and Data Engineering. vol. 20, pp. 23-31(2008)

[3]. Mitra, V., Wang, C.J., Banerjee, S.: Text Classification: A least square support vector machine approach. Journal of Applied Soft Computing. vol. 7, pp. 908--914(2007)

[4]. Seabastiani, F.: Machine Learning in Automated Text Categorization. ACM Computing Surveys. Vol. 34, pp. 1--47(2002)

[5]. Fung, G.P.C., Yu, J.X., Lu. H., Yu, P.S.: Text classification without negative example revisit. IEEE Transactions on Knowledge and Data Engineering. vol. 18, pp. 23--47(2006)

[6]. Rigutini, L.: Automatic Text Processing: Machine Learning Techniques. Ph.D. Thesis, University of Siena(2004)

[7]. Porter, M.F.: An algorithm for suffix stripping. Program, vol. 14(3), pp. 130--137(1980)

[8]. Hotho, A., Nürnberger, A., Paßß, G.: A Brief Survey of Text Mining. Journal for Computational Linguistics and Language Technology. Vol. 20, pp. 19--62(2005)

[9]. Salton, G., Wang, A., Yang, C.S.: A Vector Space Model for Automatic Indexing. Communications of the ACM, vol. 18, pp. 613--620(1975)

[10]. Hotho, A., Maedche, A., Staab, S.: Ontology-based text clustering. In: International Joint Conference on Artificial Intelligence, pp. 30--37, USA(2001)

[11]. Cavnar, W.B.: Using an N-Gram based document representation with a vector processing retrieval model. In: The Third Text Retrieval Conference (TREC-3), pp. 269--278(1994)

[12]. Milios, E., Zhang, Y., He, B., Dong, L.: Automatic term extraction and document similarity in special text corpora. In: Sixth Conference of the Pacific Association for Computational Linguistics (PACLing'03), pp. 275--284, Canada(2003)

[13]. Wei, C.P., Yang, C.C., Lin, C.M.: A Latent Semantic Indexing-based approach to multilingual document clustering. Journal of Decision Support System. Vol. 45, pp. 606--620(2008)

[14]. He, X., Cai, D., Liu, H., Ma, W.Y.: Locality Preserving Indexing for document representation. In: SIGIR, pp. 96--103(2004)

[15]. Cai, D., He, X., Zhang, W.V., Han J.: Regularized Locality Preserving Indexing via Spectral Regression. In: ACM International Conference on Information and Knowledge Management (CIKM'07), pp. 741--750, Portugal(2007)

[16]. Choudhary, B., Bhattacharyya, P.: Text clustering using Universal Networking Language representation. In: Eleventh International World Wide Web Conference(2002)

[17]. McCallum, A., Nigam, K.: A Comparison of Event Models for Naïve Bayes Text Classification. Journal of Machine Learning Research, vol. 3, pp. 1265--1287(2003)

[18]. Kim, K.B., Rim, H.C., Yook, D., Lim, H.S.: Effective Methods for improving Naïve Bayes text classifiers. In: Seventh Pacific rim International Conference on Artificial Intelligence, Springer, pp. 414--423(2002)

[19]. Tan, S.: An effective refinement strategy for KNN text classifier. Journal of Expert Systems with Applications, vol. 31, pp. 290--298(2006)

[20]. Han, E.H., Karypis, G., Kumar, V.: Text Categorization using weight adjusted K-Nearest Neighbor classification. University of Minnesota(1999)

[21]. Tan, S.: An improved centroid classifier for text categorization. Journal of Expert Systems with Applications, vol. 35, pp. 279--285(2008)

[22]. Wang, L.M., Li, X.L., Cao, C.H., Yuan, S.M.: Combining decision tree and naïve bayes for classification. Knowledge Based Systems, vol. 19, pp. 511--515(2006)

[23]. Lewis, D.D., Ringuette M.: Comparison of two learning algorithms for text categorization. In: Third Annual Symposium on Document Analysis and Information Retrieval, pp. 181--93(1994) 
[24]. Lewis, D.D., Schapire, R.E., Callan, J.P., Papka, R.: Training Algorithms for linear Text classifiers. In: Nineteenth International conference on Research and Development in Information Retrieval, pp. 289--297(1997)

[25]. Wiener, E.D., Perdersen, J.O., Weigend, A.S.: A Neural Network Approach for Topic Spotting. In: Fourth Annual Symposium on Document Analysis and Information Retrieval, pp. 317--332(1995)

[26]. Joachims, T.: Text Categorization with Support Vector Machines: Learning with many relevant features. In: Tenth European Conference on Machine Learning, pp. 137--142(1998)

[27]. Bekkerman, R., Allan, J.: Using Bigrams in Text Categorization. CIIR Technical Report, IR 408(2004)

[28]. Bernotas, M., Karklius, K., Laurutis, R., Slotkiene, A.: The peculiarities of the text document representation, using ontology and tagging-based clustering technique. Journal of Information Technology and Control. vol. 36, pp. 217--220(2007)

[29]. Dinesh, R.: POOR: Partially Occluded Object Recognizers - Some Novel Techniques. Ph.D. Thesis, University of Mysore(2006)

[30]. Bentley J. L., 1975. Multidimensional binary search trees used for associative searching. Communications of ACM, vol. 18, no. 9, pp. 509 - 517.

[31]. Kumar A., 1994. G - tree: A new datastructure for organizing multidimensional data. IEEE transactions on Knowledge and Data Engineering, vol. 6, no. 2, pp. 341 - 347.

[32]. Robinson J. T., The KDB tree: A search structure for large multidimensional dynamic indexes. Proceedings of ACM SIGMOD conference Ann Arbor, MI, pp. 10 - 18.

[33]. Dandamudi S. P and Sorenson P. G., 1985. An empirical performance comparison of some variations of the k-d tree and bd tree. Computer and Information Sciences. Vol. 14, no. 3, pp. $134-158$.

[34]. Punitha P., 2005. IARS: Image Archival and Retrieval Systems. Ph.D. Thesis, University of Mysore.

[35]. http://kdd.ics.uci.edu/databases/20newsgroups/20newsgroups.html

\section{Authors}

B S Harish obtained his B.E in Electronics and Communication, M.Tech in Networking and Internet Engineering from Visvesvaraya Technological University. He completed his Ph.D thesis entitled "Classification of Large Text Data" from University of Mysore.He is presently working as an Assistant Professor in the department of Information Science \& Engineering, SJCE, Mysore. He delivered technical talks in National and International Conferences. He has invited as a resource person to deliver a technical talks to academic staff college, University of Mysore and University of Calicut, Kerala. He is also serving as a reviewer for national, international conferences and journals. He has published more than 20 international reputed peer reviewed journals and conferences proceedings. He successfully executed AICTE-RPS project which was sanctioned by AICTE, Government of India. He also served as a secretary, CSI Mysore chapter. He is a life member of CSI and ISTE. His area of interest includes Information Retrieval, Text Mining, Pattern Recognition, Data Mining, Soft Computing and Machine Learning.

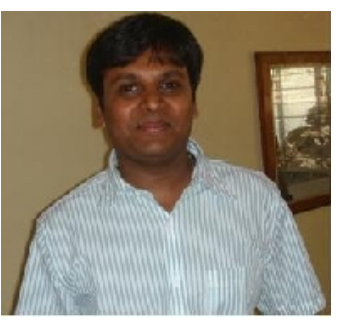


Manjunath S obtained B.Sc., and MS., degrees in Computer Science from University of Mysore, Mysore, India, respectively, in the years 2003 and 2006. Currently he is pursuing $\mathrm{Ph}$. D in video processing in University of Mysore, Mysore, India. He worked as research associate in the Department of Studies in Computer Science and currently he is a research scholar at International School of Information Management, University of Mysore. He has authored a few peer-reviewed papers in journals and conferences. His areas of research cover Image and video processing, text mining and biometrics.

D.S. Guru received B Sc, M Sc and PhD degrees in Computer Science and Technology from the University of Mysore, Mysore, India, in 1991, 1993 and 2000 respectively. He is currently an associate professor in the Department of Studies in Computer Science, University of Mysore, India. He was a fellow of BOYSCAT. He was a visiting research scientist at Michigan State University. $\mathrm{He}$ is supervising a couple of major projects sponsored by UGC, DST, Government of India. He is a life member of Indian professional bodies such as CSI, ISTE and IUPRAI. He has authored 36 journals and 145 peer reviewed conference papers at international and national levels. His area of research interest covers image retrieval, object recognition, shape analysis sign language recognition, biometrics and symbolic data analysis.
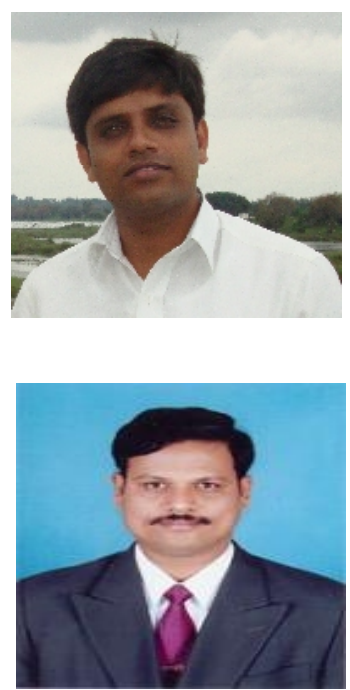